\title{
Combinatorial Algorithms for the Maximum $k$-plex Problem
}

\author{
Benjamin McClosky, Illya V. Hicks \\ Department of Computational and Applied Mathematics, Rice University, 6100 Main St - MS 134, \\ Houston, Texas 77005-1892, USA, \{bjm4@rice.edu, ivhicks@rice.edu\}
}

The maximum clique problem provides a classic framework for detecting cohesive subgraphs. However, this approach can fail to detect much of the cohesive structure in a graph. To address this issue, Seidman and Foster introduced $k$-plexes as a degree-based relaxation of graph completeness. More recently, Balasundaram et al. formulated the maximum $k$-plex problem as an integer program and designed a branch-and-cut algorithm. This paper derives a new upper bound on the cardinality of $k$-plexes and adapts combinatorial clique algorithms to find maximum $k$-plexes.

\section{Introduction}

All graphs in this paper are finite, simple, and undirected. A complete graph consists of pairwise adjacent vertices. A maximal complete subgraph defines a clique. The problem of finding maximum cardinality cliques is a classic NP-complete problem and is of fundamental importance in combinatorial optimization. The maximum clique problem has applications in ad hoc wireless networks (Chen et al. 2004), data mining (Washio and Motoda 2003), and biochemistry and genomics (Butenko and Wilhelm 2006).

Cliques also provide an intuitive approach for detecting cohesion in social networks (Festinger 1949, Luce and Perry 1949). A social network is a graph whose vertices consist of a set of actors and whose edges indicate relationships between actors. Cohesive subgroups are subsets of actors among whom there are relatively strong, direct, intense, frequent, or positive ties (Wasserman and Faust 1994). These subgroups are interesting because they facilitate the emergence of consensus among the actors (Friedkin 1984). In other words, members within a cohesive subgroup tend to exhibit homogeneity.

Despite the intuitive appeal of using cliques to model cohesion in social networks, cliques are in fact rarely appropriate for analysis of real data because the definition is too strict (Wasserman and Faust 1994). This motivates the study clique relaxations. Researchers 
have relaxed a variety of clique properties including familiarity, reachability, and robustness (Balasundaram et al. 2007). In graph theoretic terms, these properties respectively correspond to vertex degree, path length, and connectivity. This paper focuses on a degree-based relaxation known as a $k$-plex (Seidman and Foster 1978).

In order to define $k$-plexes, consider a graph $G=(V, E)$ and vertex $v \in V$. Define $N_{G}(v):=\{u \in V: u v \in E\}, \operatorname{deg}_{G}(v):=\left|N_{G}(v)\right|, \Delta(G):=\max _{v \in V} \operatorname{deg}_{G}(v)$, and $\delta(G):=$ $\min _{v \in V} \operatorname{deg}_{G}(v)$. Let $G[K]$ denote the subgraph induced by $K \subseteq V$. Define $\bar{G}=(V, \bar{E})$ to be the complement of $G$, where $e \in \bar{E} \Leftrightarrow e \notin E$. In this paper, $k \geq 1$ is a positive integer.

Definition 1 (Seidman and Foster 1978). $K \subseteq V$ induces a $k$-plex if $\delta(G[K]) \geq|K|-k$.

The term $k$-plex refers to both the set $K$ and the subgraph $G[K]$. Notice that 1-plexes are complete subgraphs. Let $\omega_{k}(G)$ denote the cardinality of a largest $k$-plex in $G$.

Definition 2 (Seidman and Foster 1978). $C \subseteq V$ induces a co-k-plex if $\Delta(G[C]) \leq k-1$.

A co- $k$-plex in $G$ is a $k$-plex in $\bar{G}$. Notice that co-1-plexes consist of pairwise nonadjacent vertices, or stable sets.

Seidman and Foster (1978) introduced $k$-plexes in the context of social networks. Balasundaram et al. (2006) provided an integer programming formulation for the maximum $k$-plex problem, derived inequalities for the $k$-plex polytope, and established the NP-completeness of the $k$-plex decision problem. McClosky and Hicks (2007) studied the co- $k$-plex polytope.

This paper develops combinatorial algorithms for finding maximum $k$-plexes. Section 2 describes heuristics for finding upper bounds on $\omega_{k}(G)$. Section 3 describes a heuristic for finding lower bounds on $\omega_{k}(G)$. Section 4 contains exact $k$-plex algorithms. Section 5 summarizes and discusses future research.

Computational Results. Sections 2-4 contain computational results obtained by conducting experiments on two classes of graphs. The first class of graphs consists entirely of DIMACS (2007) instances. The DIMACS graphs are widely considered the standard testbed for clique algorithms. The second class of graphs consists of real-life social networks. The COMP-GEOM- $t$ instances are collaboration networks for computational geometers (Beebe 2002, Jones 2002). Here, two authors are adjacent if they have jointly published more than $t$ articles. The ERDOS- $x-y$ graphs are collaboration networks of all authors with an Erdös number at most $y$ as of year $x$ (Grossman et al. 1995). Data for both collaboration networks can be downloaded from the Pajek data website (Batagelj and Mrvar 2006). 
Table 1: Test Instances

\begin{tabular}{|c|c|c|c|c|}
\hline$G$ & $|V|$ & $|E|$ & $\omega_{2}(G)$ & $\omega_{3}(G)$ \\
\hline brock200-1 & 200 & 14834 & {$[25,53]$} & - \\
\hline brock200-2 & 200 & 9876 & {$[13,24]$} & - \\
\hline brock200-4 & 200 & 13089 & {$[19,41]$} & - \\
\hline brock400-2 & 400 & 59786 & {$[27,133]$} & - \\
\hline brock400-4 & 400 & 59765 & {$[27,133]$} & - \\
\hline brock800-2 & 800 & 208166 & {$[23,253]$} & - \\
\hline brock800-4 & 800 & 207643 & {$[23,252]$} & - \\
\hline c-fat200-1 & 200 & 1534 & 12 & - \\
\hline c-fat 200-2 & 200 & 3235 & 24 & - \\
\hline c-fat $200-5$ & 200 & 8473 & 58 & - \\
\hline c-fat500-1 & 500 & 4459 & 14 & - \\
\hline c-fat500-2 & 500 & 9139 & 26 & - \\
\hline c-fat500-5 & 500 & 23191 & 64 & - \\
\hline c-fat500-10 & 500 & 46627 & 126 & - \\
\hline hamming6-2 & 64 & 1824 & 32 & - \\
\hline hamming6-4 & 64 & 704 & 6 & - \\
\hline hamming8-2 & 256 & 31616 & 128 & - \\
\hline hamming8-4 & 256 & 20864 & 16 & - \\
\hline hamming10-2 & 1024 & 518656 & {$[512,530]$} & - \\
\hline hamming10-4 & 1024 & 434176 & {$[45,153]$} & - \\
\hline johnson8-2-4 & 28 & 210 & 5 & - \\
\hline johnson8-4-4 & 70 & 1855 & 14 & - \\
\hline keller4 & 171 & 9435 & 15 & - \\
\hline MANN-a9 & 45 & 918 & 26 & - \\
\hline MANN-a27 & 378 & 70551 & 236 & - \\
\hline MANN-a45 & 1035 & 533115 & {$[662,668]$} & - \\
\hline p-hat300-1 & 300 & 10933 & {$[9,66]$} & - \\
\hline p-hat300-2 & 300 & 21928 & {$[28,85]$} & - \\
\hline p-hat300-3 & 300 & 33390 & {$[43,108]$} & - \\
\hline p-hat700-1 & 700 & 60999 & {$[10,291]$} & - \\
\hline p-hat700-2 & 700 & 121728 & {$[50,298]$} & - \\
\hline p-hat700-3 & 700 & 183010 & {$[73,311]$} & - \\
\hline $\operatorname{san} 200-0.7-2$ & 200 & 13930 & - & - \\
\hline $\operatorname{san} 200-0.9-1$ & 200 & 17910 & - & - \\
\hline COMP-GEOM-0 & 7343 & 11898 & 22 & 22 \\
\hline COMP-GEOM-1 & 7343 & 3939 & 10 & 11 \\
\hline COMP-GEOM-2 & 7343 & 1976 & 8 & 10 \\
\hline ERDOS-97-1 & 472 & 1314 & 8 & 9 \\
\hline ERDOS-98-1 & 485 & 1381 & 8 & 9 \\
\hline ERDOS-99-1 & 492 & 1417 & 8 & 9 \\
\hline ERDOS-97-2 & 5488 & 8972 & 8 & 9 \\
\hline ERDOS-98-2 & 5822 & 9505 & 8 & 9 \\
\hline ERDOS-99-2 & 6100 & 9939 & 8 & 9 \\
\hline
\end{tabular}


Table 1 contains information corresponding to the testbed. The columns $\omega_{2}(G)$ and $\omega_{3}(G)$ show the largest known $k$-plex in $G$. This data is due to Balasundaram et al. (2007). All values not specified within a range are optimal. There appears to be no known values of $\omega_{4}(G)$ for these graphs. All implementations were tested using a $2.2 \mathrm{GHz}$ Dual-Core AMD Opteron processor with 3 GB of memory.

\section{Co- $k$-plex Coloring}

This section develops an upper bound on $\omega_{k}(G)$ by generalizing the concept of graph coloring. A coloring of $G$ is a function $c_{m}: V \mapsto\{1, \ldots, m\}$ such that $c_{m}(u) \neq c_{m}(v)$ whenever $u v \in E$. The chromatic number, $\chi(G)$, of $G$ is the smallest integer $m$ for which there exists a valid coloring $c_{m}$. Notice that $c_{m}(u) \neq c_{m}(v)$ for all $u, v \in K$ whenever $K \subseteq V$ induces a complete subgraph. It follows that the chromatic number is an upper bound for $\omega(G)$. That is, $\omega(G) \leq \chi(G)$.

The goal is to find a $k$-plex analogue of this bound. To that end, suppose the co- $k$-plexes $C_{1}, \ldots, C_{m}$ partition the vertex set, and let $K$ be a maximum $k$-plex in $G$. The sets $C_{1}, \ldots, C_{m}$ define a co-k-plex coloring of $G$. Observe that

$$
\omega_{k}(G)=|K|=\sum_{i=1}^{m}\left|K \cap C_{i}\right| \leq \sum_{i=1}^{m} \omega_{k}\left(G\left[C_{i}\right]\right),
$$

where the inequality holds because $k$-plexes are closed under set inclusion (Seidman and Foster 1978). Let $\Pi$ be the set of all co- $k$-plex colorings of $G$ and define the graph invariant

$$
\chi_{k}(G):=\min \left\{\sum_{C \in \mathcal{C}} \omega_{k}(G[C]): \mathcal{C} \in \Pi\right\} .
$$

$\chi_{k}(G)$ is the co-k-plex chromatic number of $G$. Notice that $(1)$ reduces to $\omega(G) \leq \chi(G)$ when $k=1$ and $C_{1}, \ldots, C_{m}$ is an optimal coloring. It follows that $\chi_{1}(G)=\chi(G)$. Moreover, (1) and (2) together imply the bound

$$
\omega_{k}(G) \leq \chi_{k}(G)
$$

In practice, determining the exact value of $\chi_{k}(G)$ can be computationally prohibitive, so the remainder of this section is devoted to heuristics for approximating $\chi_{k}(G)$. These heuristics fall into two categories: integral and fractional. To see the distinction, let $\mathcal{I}$ be the set of all co- $k$-plexes in $G$, and let $\mathcal{I}_{v}$ denote the set of co- $k$-plexes containing $v$. Define $x(A):=\sum_{a \in A} x_{a}$. Consider the following dual pair of integer programs: 


$$
\begin{gathered}
\max \left\{x(V): x \in\{0,1\}, x(C) \leq \omega_{k}(G[C]) \text { for all } C \in \mathcal{I}\right\} \\
\min \left\{\sum_{C \in \mathcal{I}} \omega_{k}(G[C]) y_{C}: y \in\{0,1\}, y\left(\mathcal{I}_{v}\right) \geq 1 \text { for all } v \in V\right\} .
\end{gathered}
$$

The optimal objective values for $(4)$ and $(5)$ are $\omega_{k}(G)$ and $\chi_{k}(G)$, respectively. Moreover, by strong duality, the optimal objective values for the linear relaxations are equal. It follows that any feasible solution to the linear relaxation of $(5)$ produces an upper bound on $\omega_{k}(G)$. Integer Co- $k$-plex Coloring Heuristics (ICCH) find feasible solutions to (5). Fractional Co- $k$ plex Coloring Heuristics (FCCH) find feasible solutions to the linear relaxation of (5). Both heuristics make use of the following lemmas which provide analytic bounds on $\omega_{k}(G)$.

Lemma 1. Every graph $G$ satisfies $\omega_{k}(G) \leq \Delta(G)+k$.

Proof. If there exists a $k$-plex $K$ in $G$ such that $|K|>\Delta(G)+k$, then $\operatorname{deg}_{G[K]}(v) \geq|K|-k>$ $\Delta(G)$ for all $v \in K$, a contradiction.

Lemma 2. Given a graph $G$ and an integer $m \geq 1$, let $a_{m}$ denote the number of vertices $v \in V$ such that $\operatorname{deg}_{G}(v) \geq m$. If $j:=\max \left\{m: a_{m} \geq k+m\right\}$, then $\omega_{k}(G) \leq k+j$.

Proof. Suppose $G$ contains a $k$-plex $K$ such that $|K| \geq k+j+1$. By definition of $k$-plex,

$$
\operatorname{deg}_{G[K]}(v) \geq|K|-k \geq j+1 \quad \text { for all } v \in K
$$

In other words, $K$ contains at least $k+j+1$ vertices $v$ such that $\operatorname{de} g_{G[K]}(v) \geq j+1$. It follows that $a_{j+1} \geq k+j+1$, contradicting the definition of $j$.

Lemma 3 (Balasundaram et al. 2006). If $G$ is a co-k-plex, then $\omega_{k}(G) \leq 2 k-2+k \bmod 2$.

Figure 1 shows an ICCH. Line 4 stores the degree of every vertex in $C_{m}$. Line 7 uses Lemmas 1,2 , and 3 to bound $\omega_{k}\left(G\left[C_{i}\right]\right)$. Lines $3,4,6$, and 7 can each be accomplished in linear time using an adjacency matrix. It follows that this ICCH is an $\mathcal{O}\left(|V|^{2}\right)$ algorithm. Table 2 contains computational results obtained by running the function ICCH on the benchmark graphs with an arbitrary initial vertex ordering.

The FCCH adapts the fractional coloring procedure of Balas and Xue (1996). More precisely, the FCCH constructs a set of co- $k$-plexes $C_{1}, \ldots, C_{h} \subseteq V$ such that, after $p$ iterations, 
Table 2: ICCH Results

\begin{tabular}{lcccccc}
\hline \multicolumn{1}{c}{$G$} & $\chi_{2}(G)$ & seconds & $\chi_{3}(G)$ & seconds & $\chi_{4}(G)$ & seconds \\
\hline brock200-1 & 93 & 0.0 & 151 & 0.0 & 169 & 0.0 \\
brock200-2 & 55 & 0.0 & 95 & 0.0 & 118 & 0.0 \\
brock200-4 & 78 & 0.0 & 131 & 0.0 & 151 & 0.0 \\
brock400-2 & 172 & 0.1 & 285 & 0.1 & 332 & 0.1 \\
brock400-4 & 168 & 0.1 & 286 & 0.1 & 330 & 0.1 \\
brock800-2 & 248 & 0.3 & 442 & 0.3 & 570 & 0.3 \\
brock800-4 & 247 & 0.3 & 440 & 0.3 & 557 & 0.3 \\
c-fat200-1 & 18 & 0.0 & 20 & 0.0 & 21 & 0.0 \\
c-fat200-2 & 34 & 0.0 & 37 & 0.0 & 38 & 0.0 \\
c-fat200-5 & 82 & 0.0 & 89 & 0.0 & 90 & 0.0 \\
c-fat500-1 & 19 & 0.0 & 23 & 0.0 & 24 & 0.0 \\
c-fat500-2 & 36 & 0.0 & 41 & 0.0 & 42 & 0.0 \\
c-fat500-5 & 85 & 0.1 & 98 & 0.0 & 99 & 0.0 \\
c-fat500-10 & 172 & 0.1 & 191 & 0.1 & 192 & 0.1 \\
hamming6-2 & $32 *$ & 0.0 & 48 & 0.0 & 56 & 0.0 \\
hamming6-4 & 8 & 0.0 & 12 & 0.0 & 16 & 0.0 \\
hamming8-2 & $128^{*}$ & 0.0 & 192 & 0.0 & 224 & 0.0 \\
hamming8-4 & 32 & 0.0 & 48 & 0.0 & 64 & 0.0 \\
hamming10-2 & $512^{*}$ & 0.7 & 768 & 0.7 & 896 & 0.7 \\
hamming10-4 & 128 & 0.5 & 192 & 0.5 & 256 & 0.5 \\
johnson8-2-4 & 12 & 0.0 & 16 & 0.0 & 19 & 0.0 \\
johnson8-4-4 & 28 & 0.0 & 42 & 0.0 & 48 & 0.0 \\
keller4 & 54 & 0.0 & 100 & 0.0 & 128 & 0.0 \\
MANN-a9 & 38 & 0.0 & 44 & 0.0 & 45 & 0.0 \\
MANN-a27 & 324 & 0.1 & 369 & 0.1 & 378 & 0.1 \\
MANN-a45 & 833 & 1.0 & 1032 & 0.8 & 1035 & 0.8 \\
p-hat300-1 & 39 & 0.0 & 69 & 0.0 & 90 & 0.0 \\
p-hat300-2 & 76 & 0.0 & 135 & 0.0 & 173 & 0.0 \\
p-hat300-3 & 129 & 0.0 & 210 & 0.0 & 245 & 0.0 \\
p-hat700-1 & 76 & 0.1 & 135 & 0.1 & 184 & 0.1 \\
p-hat700-2 & 165 & 0.2 & 289 & 0.1 & 375 & 0.2 \\
p-hat700-3 & 267 & 0.3 & 451 & 0.2 & 556 & 0.2 \\
san200-0.7-2 & 105 & 0.0 & 147 & 0.0 & 159 & 0.0 \\
san200-0.9-1 & 133 & 0.0 & 184 & 0.0 & 193 & 0.0 \\
COMP-GEOM-0 & 29 & 10 & 48 & 10 & 50 & 11 \\
COMP-GEOM-1 & 22 & 9.8 & 37 & 10 & 37 & 11 \\
COMP-GEOM-2 & 16 & 10 & 28 & 11 & 27 & 10 \\
ERDOS971 & 20 & 0.1 & 32 & 0.1 & 31 & 0.1 \\
ERDOS972 & 19 & 5.9 & 36 & 4.9 & 36 & 5.1 \\
ERDOS981 & 18 & 0.1 & 33 & 0.1 & 32 & 0.1 \\
ERDOS982 & 20 & 5.8 & 36 & 5.7 & 37 & 5.9 \\
ERDOS991 & 18 & 0.0 & 34 & 0.1 & 34 & 0.1 \\
ERDOS992 & 20 & 6.3 & 38 & 6.5 & 38 & 6.6 \\
\hline * 0.0 & & & \\
*aptima & & & & & & \\
\hline
\end{tabular}

* optimal 
every vertex belongs to exactly $p$ distinct co- $k$-plex sets. These co- $k$-plexes combine to form a feasible solution to the linear relaxation of (5) as follows:

$$
\bar{y}:=\frac{1}{p} \sum_{i=1}^{h} y_{C_{i}} .
$$

The feasibility of $\bar{y}$ implies that

$$
\omega_{k}(G) \leq \frac{1}{p} \sum_{i=1}^{h} \omega_{k}\left(G\left[C_{i}\right]\right) \bar{y}_{C_{i}} .
$$

Figure 2 shows the FCCH. The set $\mathcal{C}$ consists of the co- $k$-plexes $C_{1}, \ldots, C_{h}$. At every iteration, each vertex is either added to an existing $C_{i} \in \mathcal{C}$ in Line 7 or to a new partition set in Line 10. By Line 12, every vertex belongs to exactly $p$ partition sets, so $t_{n e w}$ is a valid upper bound on $\omega_{k}(G)$. The FCCH termination condition can be inefficient, so the number of iterations and the number partition sets in $\mathcal{C}$ are both required to be $\mathcal{O}(|V|)$.

Theorem 1. If the number of iterations and the number of partition sets are $\mathcal{O}(|V|)$, then FCCH can be executed in $\mathcal{O}\left(|V|^{4}\right)$ time.

Proof. At every iteration, for each vertex $v \in V$, the algorithm tests if $C_{i} \cup\{v\}$ is a co- $k$-plex. This can be done by counting the number of $u \in N_{G}(v) \cap C_{i}$, which requires $\mathcal{O}(|V|)$ time. Since there are $\mathcal{O}(|V|)$ partition sets, there can be $\mathcal{O}\left(|V|^{2}\right)$ possible pairs $\left(C_{i}, v\right)$. Thus, after $\mathcal{O}(|V|)$ iterations, this step has complexity $\mathcal{O}\left(|V|^{4}\right)$. Lines 2 and 10 execute the $\mathcal{O}\left(|V|^{2}\right)$ ICCH algorithm. Since there are at most $\mathcal{O}(|V|)$ iterations, these steps have complexity $\mathcal{O}\left(|V|^{3}\right)$. All other operations contribute $\mathcal{O}\left(|V|^{2}\right)$ to the complexity. Therefore, the overall complexity of FCCH is $\mathcal{O}\left(|V|^{4}\right)$.

\section{function $\operatorname{ICCH}(V)$}

1. $C_{i}=\varnothing$ for $1 \leq i \leq|V|$

2. for all $u \in V$

3. $m=\min \left\{i: C_{i} \cup\{u\}\right.$ is a co-k-plex in $\left.G\right\}$

4. $C_{m}=C_{m} \cup\{u\}$

5. end

6. Compute $j_{i}:=\max \left\{m: a_{m} \geq k+m\right\}$ for each $C_{i}$

7. $\quad$ bound $=\sum_{i=1}^{|V|} \min \left\{2 k-2+k \bmod 2, k+j_{i}, \Delta\left(G\left[C_{i}\right]\right)+k,\left|C_{i}\right|\right\}$

8. return bound

Figure 1: An Integer Co- $k$-plex Coloring Heuristic 
Table 3: FCCH Results

\begin{tabular}{|c|c|c|c|c|c|c|}
\hline$G$ & $\chi_{2}(G)$ & seconds & $\chi_{3}(G)$ & seconds & $\chi_{4}(G)$ & seconds \\
\hline brock200-1 & 82 & 0.0 & 139 & 0.1 & 164 & 0.0 \\
\hline brock200-2 & 48 & 0.0 & 89 & 0.0 & 118 & 0.0 \\
\hline brock200-4 & 68 & 0.0 & 122 & 0.0 & 151 & 0.0 \\
\hline brock400-2 & 151 & 0.2 & 267 & 0.2 & 320 & 0.2 \\
\hline brock400-4 & 151 & 0.2 & 265 & 0.3 & 320 & 0.1 \\
\hline brock800-2 & 221 & 1.4 & 401 & 1.7 & 535 & 1.3 \\
\hline brock800-4 & 223 & 2.4 & 410 & 0.8 & 537 & 1.2 \\
\hline c-fat200-1 & 16 & 0.0 & 20 & 0.0 & 21 & 0.0 \\
\hline c-fat $200-2$ & 30 & 0.0 & 37 & 0.0 & 38 & 0.0 \\
\hline c-fat $200-5$ & 76 & 0.0 & 89 & 0.0 & 90 & 0.0 \\
\hline c-fat $500-1$ & 18 & 0.1 & 23 & 0.0 & 24 & 0.0 \\
\hline c-fat $500-2$ & 33 & 0.1 & 41 & 0.0 & 42 & 0.0 \\
\hline c-fat $500-5$ & 82 & 0.2 & 98 & 0.1 & 99 & 0.1 \\
\hline c-fat500-10 & 166 & 0.4 & 191 & 0.1 & 192 & 0.2 \\
\hline hamming6-2 & $32^{*}$ & 0.0 & 48 & 0.0 & 56 & 0.0 \\
\hline hamming6-4 & 8 & 0.0 & 12 & 0.0 & 16 & 0.0 \\
\hline hamming8-2 & $128^{*}$ & 0.0 & 192 & 0.0 & 224 & 0.1 \\
\hline hamming8-4 & 32 & 0.0 & 48 & 0.0 & 64 & 0.0 \\
\hline hamming10-2 & $512^{*}$ & 1.3 & 768 & 1.3 & 896 & 2.0 \\
\hline hamming10-4 & 128 & 0.7 & 192 & 0.6 & 256 & 0.6 \\
\hline johnson8-2-4 & 10 & 0.0 & 16 & 0.0 & 18 & 0.0 \\
\hline johnson8-4-4 & 28 & 0.0 & 42 & 0.0 & 46 & 0.0 \\
\hline keller4 & 45 & 0.0 & 88 & 0.0 & 113 & 0.0 \\
\hline MANN-a9 & 36 & 0.0 & 44 & 0.0 & 45 & 0.0 \\
\hline MANN-a27 & 321 & 0.2 & 366 & 0.1 & 378 & 0.1 \\
\hline MANN-a45 & 803 & 5.9 & 1028 & 1.8 & 1035 & 1.1 \\
\hline p-hat300-1 & 34 & 0.0 & 62 & 0.0 & 85 & 0.0 \\
\hline p-hat300-2 & 71 & 0.1 & 129 & 0.0 & 161 & 0.1 \\
\hline p-hat300-3 & 115 & 0.2 & 201 & 0.1 & 240 & 0.1 \\
\hline p-hat700-1 & 68 & 0.3 & 123 & 0.3 & 168 & 0.3 \\
\hline p-hat700-2 & 146 & 0.7 & 272 & 0.3 & 349 & 0.5 \\
\hline p-hat700-3 & 243 & 1.4 & 428 & 0.8 & 532 & 0.6 \\
\hline $\operatorname{san} 200-0.7-2$ & 79 & 0.0 & 140 & 0.0 & 159 & 0.0 \\
\hline $\operatorname{san} 200-0.9-1$ & 127 & 0.0 & 177 & 0.1 & 191 & 0.1 \\
\hline COMP-GEOM-0 & 23 & 18 & 44 & 18 & 43 & 18 \\
\hline COMP-GEOM-1 & 15 & 17 & 28 & 17 & 30 & 16 \\
\hline COMP-GEOM-2 & 12 & 17 & 22 & 17 & 23 & 17 \\
\hline ERDOS971 & 14 & 0.0 & 25 & 0.0 & 29 & 0.1 \\
\hline ERDOS972 & 14 & 9.6 & 28 & 8.7 & 30 & 8.8 \\
\hline ERDOS981 & 12 & 0.1 & 25 & 0.1 & 28 & 0.1 \\
\hline ERDOS982 & 14 & 9.9 & 30 & 9.9 & 29 & 10 \\
\hline ERDOS991 & 12 & 0.1 & 25 & 0.0 & 28 & 0.1 \\
\hline ERDOS992 & 14 & 11 & 28 & 11 & 30 & 11 \\
\hline
\end{tabular}

* optimal 


\section{function $\mathbf{F C C H}(V)$}

1. $t_{\text {old }}=\infty ; p=1$

2. $\quad t_{\text {new }}=\mathbf{I C C H}(V)$; store the partition sets in $\mathcal{C}$

3. $\quad$ while $t_{\text {new }}<t_{\text {old }}$

4. $U=V ; t_{\text {old }}=t_{\text {new }} ; \quad p=p+1$

5. for all $v \in U$

6. $\quad$ if $\exists C_{i} \in \mathcal{C}$ such that $v \notin C_{i}$ and $C_{i} \cup\{v\}$ is a co- $k$-plex

7. $\quad C_{i}=C_{i} \cup\{v\} ; U=U \backslash\{v\}$

8. $\quad$ end

9. $\quad$ end

10. $\quad \mathbf{I C C H}(U)$; append new partition sets in $\mathcal{C}$

11. Compute $j_{i}:=\max \left\{m: a_{m} \geq k+m\right\}$ for each $C_{i} \in \mathcal{C}$

12. $t_{\text {new }}=\frac{1}{p} \cdot \sum_{C_{i} \in \mathcal{C}} \min \left\{2 k-2+k \bmod 2, k+j_{i}, \Delta\left(G\left[C_{i}\right]\right)+k,\left|C_{i}\right|\right\}$

13. end

14. return $t_{\text {old }}$

Figure 2: A Fractional Co- $k$-plex Coloring Heuristic

Table 3 contains computational results obtained by running the function FCCH on the benchmark graphs with an arbitrary initial vertex. The bound on the number of iterations was set to $5 \cdot|V|$.

\section{A $k$-plex Heuristic}

This section describes a heuristic for finding maximum $k$-plexes. Feasible $k$-plexes provide lower bounds on $\omega_{k}(G)$. The heuristic indirectly searches for cohesive subgraphs in $G$ and extends them to maximal $k$-plexes.

There has been extensive research on heuristics for finding large complete subgraphs (Busygin et al. 2002, Feo and Resende 2005, Gendreau 1993, Marchiori 2002). A typical combinatorial heuristic systematically searches a set of neighborhoods in the feasible solution space for local optima (Hansen et al. 2004). When a local optimum is obtained, it is compared to the incumbent solution and stored if necessary. The heuristic then continues searching in other neighborhoods. Obviously, the solution quality heavily depends on both the choice of neighborhoods and the local search method.

Recall that if $\mathcal{I}_{G}$ denotes the set of all complete subgraphs in $G$, then $\mathcal{I}_{G}$ also denotes the set of all stable sets in $\bar{G}$. The remainder of this section focuses on finding stable sets in $\bar{G}$ which are extended to maximal $k$-plexes in $G$. This approach is valid because every element in $\mathcal{I}_{G}$ is extendible to a maximal $k$-plex in $G$. Without loss of generality, assume $G$ 


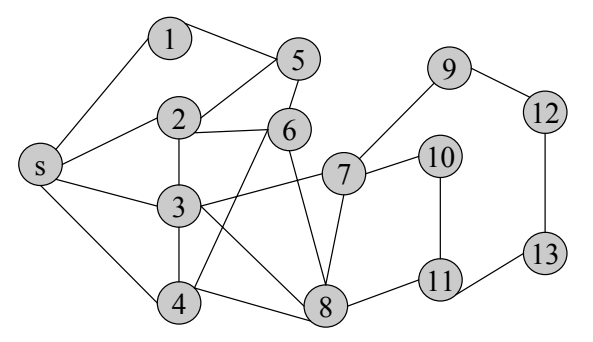

Figure 3: $\bar{H}$ with root $s$.

is connected. For if not, simply run the heuristic on each component.

For $u, v \in V$, let $d(u, v)$ be the length of a shortest path from $u$ to $v$ in $G$. The concept of neighborhood is based on the parity of shortest path lengths from some root node $s$. Given a root $s \in V$, define the following sets:

$$
K_{0}:=\{v \in V \mid d(s, v) \text { even }\} \quad \text { and } \quad K_{1}:=\{v \in V \mid d(s, v) \text { odd }\} .
$$

For example, consider the search for $k$-plexes in some graph $H$, and suppose that $\bar{H}$ is shown in Figure 3. The vertex set $V(H)$ partitions into the sets $K_{0}=\{s, 5,6,7,8,12,13\}$ and $K_{1}=\{1,2,3,4,9,10,11\}$. For $i \in\{0,1\}$, notice that $u, v \in K_{i}$ and $u v \in E(\bar{H})$ together imply $d(u, s)=d(v, s)$. Otherwise, $d(u, s)$ and $d(v, s)$ would have different parities. Therefore, for every $v \in K_{i}$,

$$
N_{\bar{H}}(v) \cap\left\{u \in K_{i} \backslash\{v\}: d(u, s) \neq d(v, s)\right\}=\varnothing .
$$

Hopefully, this property causes $K_{i}$ to contain large stable sets.

Now $K_{i} \notin \mathcal{I}_{H}$ in general, but there will typically exist many subsets $K_{i}^{\prime} \subseteq K_{i}$ such that $K_{i}^{\prime} \in \mathcal{I}_{H}$. In order to examine a variety of these subsets, construct elements in $\mathcal{I}_{H}$ from $K_{i}$ by removing one end of every edge in $\bar{H}\left[K_{i}\right]$. To determine which end of edge $u v \in E\left(\bar{H}\left[K_{i}\right]\right)$ to remove, always apply exactly one of the following rules:

Rule 1. If $\operatorname{deg}_{\bar{H}\left[K_{i}\right]}(v) \leq \operatorname{deg}_{\bar{H}\left[K_{i}\right]}(u)$, remove $u$. Otherwise, remove $v$.

Rule 2. If $\operatorname{deg}_{\bar{H}}(v) \leq \operatorname{deg}_{\bar{H}}(u)$, remove $u$. Otherwise, remove $v$.

Rule 3. Always remove $v$.

Rule 4. Always remove $u$.

Let $K_{i}^{j}$ be the subset obtained from $K_{i}$ be applying Rule $j$ to every edge in $E\left(\bar{H}\left[K_{i}\right]\right)$. Rules 1 and 2 are greedy metrics. Rules 3 and 4 are included to diversify the search space.

Now extend each set $K_{i}^{j}$ to a maximal $k$-plex in $H$. All $k$-plexes that can be constructed from a set $K_{i}$ in this way constitute a neighborhood. Therefore, the search space is essentially 


\section{function $\operatorname{lbound}(\mathcal{R})$}

1. for all $s \in \mathcal{R}$

2. define $K_{0}$ and $K_{1}$ with respect to root $s$

3. construct sets $K_{i}^{j} \subseteq K_{i}$

4. extend sets $K_{i}^{j}$ to maximal $k$-plexes in $H$

5. $\quad$ for all $j$ and $i$

6. $\quad \operatorname{kick}\left(K_{i}^{j}\right)$

7. end

8. update incumbent $I$ if necessary

9. end

\section{function $\operatorname{kick}(K)$}

10. construct set $S:=\left\{v \in V \backslash K:\left|N_{\bar{H}}(v) \cap K\right| \leq 1\right\}$

11. let $K=K \cup S$

12. construct sets $K^{j} \subseteq K$

13. extend sets $K^{j}$ to maximal $k$-plexes in $H$

14. end

Figure 4: $k$-plex heuristic.

a function of the root nodes, and specifying a set of neighborhoods is equivalent to specifying a set of root nodes $\mathcal{R}$. The $k$-plex heuristic is shown in Figure 4 . The incumbent solution $I$ is initially empty and stored as a global variable.

To find a $k$-plex in $H$, arbitrarily choose a set of vertices to define $\mathcal{R}$. Line 2 builds a breadth-first-search tree in $\bar{H}$ rooted at $s$ to determine $d(v, s)$ for all $v \in V$. The breadthfirst-search tree is also used to define $\operatorname{deg}_{\bar{H}\left[K_{i}\right]}(v)$ for all $v$. Line 3 applies Rules 1-4, and Line 4 uses a greedy heuristic. Line 6 passes the sets $K_{i}^{j}$ to the new function kick. Its purpose is to help the heuristic escape local optima. Line 12 uses Rules 1-4 for each input set $K$. Figure 4 is a basic $k$-plex heuristic. Table 4 contains computational results obtained by running lbound on the benchmark graphs. LB1 corresponds to choosing an arbitrary

set of $\frac{|V|}{40}$ vertices to define $\mathcal{R}$. LB2 corresponds to setting $\mathcal{R}=V$. The heuristics terminate after an hour time limit.

\section{Exact Algorithms}

This section describes exact algorithms for finding maximum $k$-plexes in a graph $G=$ $(V, E)$. The first type is based on a standard clique algorithm (Applegate and Johnson 1993, Carraghan and Pardalos 1990). The second type adapts an algorithm of Östergård (2002). 
Table 4: lbound Results

\begin{tabular}{|c|c|c|c|c|c|c|c|c|c|c|c|c|}
\hline & LB1 & & LB2 & & LB1 & & LB2 & & LB1 & & LB2 & \\
\hline$G$ & $\omega_{2}(G)$ & sec. & $\omega_{2}(G)$ & sec. & $\omega_{3}(G)$ & sec. & $\omega_{3}(G)$ & sec. & $\omega_{4}(G)$ & sec. & $\omega_{4}(G)$ & sec. \\
\hline brock200-1 & 25 & 1 & 25 & 3 & 27 & 1 & 28 & 3 & 31 & 1 & 32 & 3 \\
\hline brock200-2 & 12 & 1 & $13^{*}$ & 5 & 15 & 1 & 15 & 6 & 17 & 1 & 17 & 5 \\
\hline brock200-4 & 18 & 1 & 19 & 4 & 22 & 1 & 23 & 4 & 24 & 1 & 25 & 4 \\
\hline brock400-2 & 27 & 2 & 28 & 23 & 31 & 2 & 32 & 23 & 35 & 2 & 36 & 23 \\
\hline brock400-4 & 33 & 2 & 33 & 23 & 33 & 2 & 33 & 23 & 36 & 2 & 37 & 23 \\
\hline brock800-2 & 22 & 15 & 23 & 299 & 26 & 15 & 26 & 298 & 29 & 15 & 30 & 299 \\
\hline brock $800-4$ & 22 & 15 & 23 & 301 & 25 & 15 & 26 & 301 & 29 & 15 & 30 & 301 \\
\hline c-fat200-1 & $12^{*}$ & 2 & $12^{*}$ & 10 & $12^{*}$ & 2 & $12^{*}$ & 10 & $12^{*}$ & 2 & $12^{*}$ & 10 \\
\hline c-fat $200-2$ & $24^{*}$ & 2 & $24^{*}$ & 9 & $24^{*}$ & 2 & $24^{*}$ & 9 & $24^{*}$ & 2 & $24^{*}$ & 10 \\
\hline c-fat $200-5$ & $58^{*}$ & 2 & $58^{*}$ & 7 & $58^{*}$ & 2 & $58^{*}$ & 7 & $58^{*}$ & 2 & $58^{*}$ & 7 \\
\hline c-fat500-1 & $14^{*}$ & 20 & $14^{*}$ & 225 & $14^{*}$ & 19 & $14^{*}$ & 226 & $14^{*}$ & 19 & $14^{*}$ & 226 \\
\hline c-fat500-2 & $26^{*}$ & 19 & $26^{*}$ & 210 & $26^{*}$ & 18 & $26^{*}$ & 210 & $26^{*}$ & 19 & $26^{*}$ & 212 \\
\hline c-fat500-5 & $64^{*}$ & 16 & $64^{*}$ & 191 & $64^{*}$ & 16 & $64^{*}$ & 185 & $64^{*}$ & 16 & $64^{*}$ & 194 \\
\hline c-fat500-10 & $126^{*}$ & 13 & $126^{*}$ & 146 & $126^{*}$ & 13 & $126^{*}$ & 150 & $126^{*}$ & 13 & $126^{*}$ & 152 \\
\hline hamming6-2 & $32^{*}$ & 0 & $32^{*}$ & 0 & $32^{*}$ & 0 & $32^{*}$ & 0 & 32 & 0 & 32 & 0 \\
\hline hamming6-4 & 4 & 0 & 4 & 0 & $8^{*}$ & 0 & $8^{*}$ & 0 & 8 & 0 & 8 & 0 \\
\hline hamming8-2 & $128^{*}$ & 0 & $128^{*}$ & 2 & $128^{*}$ & 0 & $128^{*}$ & 2 & 128 & 0 & 128 & 2 \\
\hline hamming8-4 & $16^{*}$ & 1 & $16^{*}$ & 8 & 16 & 1 & 16 & 8 & 16 & 1 & 16 & 8 \\
\hline hamming10-2 & $512^{*}$ & 3 & $512^{*}$ & 65 & 512 & 3 & 512 & 67 & 512 & 3 & 512 & 74 \\
\hline hamming10-4 & 43 & 12 & 43 & 281 & 64 & 12 & 64 & 288 & 63 & 12 & 64 & 297 \\
\hline johnson8-2-4 & 4 & 0 & 4 & 0 & $8^{*}$ & 0 & $8^{*}$ & 0 & $9^{*}$ & 0 & $9^{*}$ & 0 \\
\hline johnson8-4-4 & 14 & 0 & 14 & 0 & 14 & 0 & 14 & 0 & 14 & 0 & 14 & 0 \\
\hline keller4 & $15^{*}$ & 1 & $15^{*}$ & 3 & 18 & 1 & 18 & 2 & 20 & 1 & 20 & 2 \\
\hline MANN-a9 & 22 & 0 & 22 & 0 & 30 & 0 & 30 & 0 & $36^{*}$ & 0 & $36^{*}$ & 0 \\
\hline MANN-a27 & 218 & 2 & 218 & 14 & 258 & 3 & 260 & 29 & 250 & 2 & 257 & 17 \\
\hline MANN-a45 & 646 & 35 & 646 & 859 & 762 & 76 & 762 & 1748 & 756 & 21 & 756 & 540 \\
\hline p-hat300-1 & 9 & 4 & 9 & 28 & 11 & 4 & 11 & 28 & 12 & 4 & 13 & 28 \\
\hline p-hat300-2 & 30 & 3 & 30 & 20 & 34 & 3 & 34 & 19 & 39 & 3 & 39 & 20 \\
\hline p-hat300-3 & 42 & 1 & 43 & 10 & 49 & 1 & 49 & 10 & 53 & 2 & 55 & 11 \\
\hline p-hat700-1 & 10 & 33 & 12 & 537 & 13 & 33 & 14 & 555 & 16 & 32 & 16 & 529 \\
\hline p-hat700-2 & 50 & 19 & 51 & 316 & 58 & 19 & 58 & 320 & 65 & 19 & 66 & 321 \\
\hline p-hat700-3 & 70 & 8 & 71 & 140 & 82 & 9 & 84 & 140 & 92 & 9 & 95 & 141 \\
\hline $\operatorname{san} 200-0.7-2$ & 26 & 1 & 26 & 3 & 36 & 1 & 36 & 4 & 48 & 1 & 48 & 4 \\
\hline $\operatorname{san} 200-0.9-1$ & 90 & 0 & 90 & 2 & $125^{*}$ & 0 & $125^{*}$ & 2 & 125 & 0 & 125 & 2 \\
\hline COMP-GEOM-0 & 16 & 1439 & $22^{*}$ & 3600 & 16 & 1551 & $22^{*}$ & 3600 & 16 & 1545 & 22 & 3600 \\
\hline COMP-GEOM-1 & 8 & 1489 & $10^{*}$ & 3600 & 8 & 1597 & $11^{*}$ & 3600 & 8 & 1531 & 11 & 3600 \\
\hline COMP-GEOM-2 & 8 & 1529 & $8^{*}$ & 3600 & 8 & 1543 & 8 & 3600 & 8 & 1468 & 8 & 3600 \\
\hline ERDOS971 & $8^{*}$ & 18 & $8^{*}$ & 3600 & $9^{*}$ & 17 & $9^{*}$ & 3600 & 10 & 17 & 10 & 3600 \\
\hline ERDOS972 & $8^{*}$ & 2506 & $8^{*}$ & 3506 & $9^{*}$ & 2433 & $9^{*}$ & 3600 & 10 & 2483 & 10 & 3600 \\
\hline ERDOS981 & $8^{*}$ & 17 & $8^{*}$ & 3600 & $9^{*}$ & 17 & $9^{*}$ & 3600 & 10 & 17 & 10 & 3600 \\
\hline ERDOS982 & $8^{*}$ & 2473 & $8^{*}$ & 3600 & $9^{*}$ & 2698 & $9^{*}$ & 3600 & 10 & 2632 & 10 & 3600 \\
\hline ERDOS991 & $8^{*}$ & 18 & $8^{*}$ & 3600 & $9^{*}$ & 18 & $9^{*}$ & 3600 & 10 & 18 & 10 & 3600 \\
\hline ERDOS992 & $8^{*}$ & 2103 & $8^{*}$ & 3600 & $9^{*}$ & 2319 & $9^{*}$ & 3600 & 10 & 2746 & 10 & 3600 \\
\hline
\end{tabular}




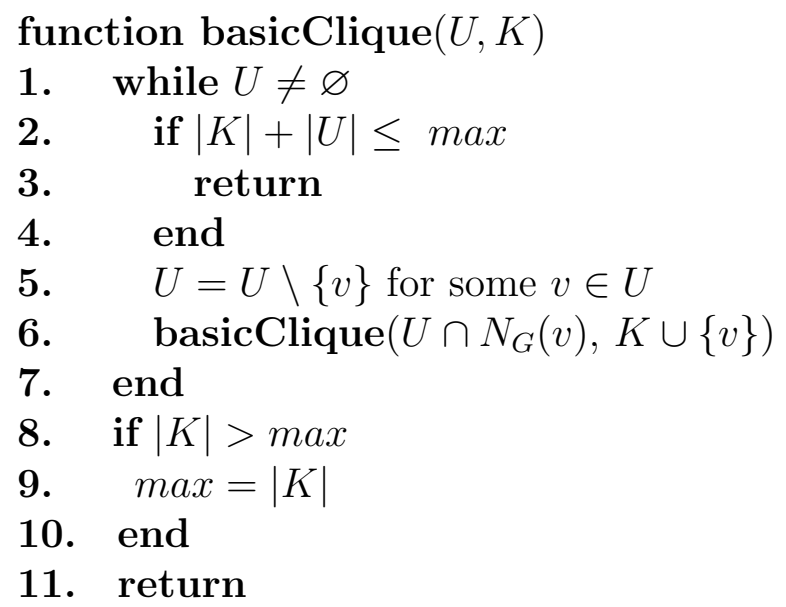

Figure 5: Basic Clique Algorithm

\subsection{Algorithm Type 1}

Consider the standard clique algorithm shown in Figure 5. At any point, the algorithm is constructing a complete graph $K$. The candidate set, $U \subseteq V \backslash K$, contains all vertices $v$ such that $K \cup\{v\}$ is complete. In other words, $U:=\bigcap_{v \in K} N_{G}(v)$. The global variable max stores the cardinality of the largest clique found. To find a maximum clique, initialize $\max =0$ and make the function call basicClique $(V, \varnothing)$.

This clique algorithm generalizes to find maximum $k$-plexes. The main difference is that the candidate set $U$ for a $k$-plex $K$ is no longer $\bigcap_{v \in K} N_{G}(v)$. It is now defined as

$$
U:=\{v \in V \backslash K: K \cup\{v\} \text { is a } k \text {-plex }\} .
$$

Figure 6 shows the basic $k$-plex algorithm. To find a maximum $k$-plex, initialize max $=0$ and make the function call basicPlex $(V, \varnothing)$. Table 5 contains computational results obtained by running basicPlex on the benchmark graphs with a one hour time limit.

Without Lines 2-4, the clique algorithm examines every clique in $G$. Recall that $G$ can contain an exponential, with respect to $|V|$, number of cliques (Moon and Moser 1965). Lines 2-4 attempt to avoid enumeration of an exponential number of subgraphs. This is known as pruning the search tree. Although there may exist graphs which require the enumeration of an exponential number of cliques, pruning can reduce the runtime.

The basic clique algorithm has many variants (Régin 2003, Sewell 1998, Tomita and Seki 2003, Wood 1997). Many researchers focus on improving the pruning strategy using the coloring bound. A coloring heuristic provides an upper bound on $\omega(G[U])$ and has the 
Table 5: basicPlex Results

\begin{tabular}{|c|c|c|c|c|c|c|c|c|c|}
\hline$G$ & $\omega_{2}(G)$ & seconds & BBN & $\omega_{3}(G)$ & seconds & BBN & $\omega_{4}(G)$ & seconds & BBN \\
\hline brock200-1 & 25 & $\geq 3600$ & 172822699 & 28 & $\geq 3600$ & 182056437 & 31 & $\geq 3600$ & 180633250 \\
\hline brock200-2 & $13^{*}$ & 166 & 9759381 & 15 & $\geq 3600$ & 199178608 & 17 & $\geq 3600$ & 192281927 \\
\hline brock200-4 & 20 & $\geq 3600$ & 193074734 & 22 & $\geq 3600$ & 199289654 & 25 & $\geq 3600$ & 193677120 \\
\hline brock400-2 & 27 & $\geq 3600$ & 169761253 & 31 & $\geq 3600$ & 162264447 & 34 & $\geq 3600$ & 155153487 \\
\hline brock $400-4$ & 27 & $\geq 3600$ & 160979618 & 32 & $\geq 3600$ & 146807899 & 36 & $\geq 3600$ & 145283598 \\
\hline brock800-2 & 23 & $\geq 3600$ & 134201916 & 25 & $\geq 3600$ & 139748190 & 28 & $\geq 3600$ & 124918468 \\
\hline brock800-4 & 23 & $\geq 3600$ & 133857528 & 24 & $\geq 3600$ & 144247348 & 27 & $\geq 3600$ & 127834010 \\
\hline c-fat200-1 & $12^{*}$ & 0 & 975 & $12^{*}$ & 4 & 58324 & $12^{*}$ & 170 & 2025883 \\
\hline c-fat200-2 & $24^{*}$ & 0 & 7308 & $24^{*}$ & 5 & 115832 & $24^{*}$ & 226 & 3827925 \\
\hline c-fat $200-5$ & $58^{*}$ & 3112 & 86024721 & 58 & $\geq 3600$ & 104935293 & 58 & $\geq 3600$ & 108945815 \\
\hline c-fat500-1 & $14^{*}$ & 1 & 2712 & $14^{*}$ & 115 & 364617 & 14 & $\geq 3600$ & 6098258 \\
\hline c-fat $500-2$ & $26^{*}$ & 1 & 31068 & $26^{*}$ & 126 & 818322 & 26 & $\geq 3600$ & 14272751 \\
\hline c-fat $500-5$ & 64 & $\geq 3600$ & 84968699 & 64 & $\geq 3600$ & 94102915 & 64 & $\geq 3600$ & 92359122 \\
\hline c-fat500-10 & 126 & $\geq 3600$ & 39813170 & 126 & $\geq 3600$ & 45937780 & 126 & $\geq 3600$ & 45046647 \\
\hline hamming6-2 & $32^{*}$ & 506 & 26461612 & 32 & $\geq 3600$ & 244753572 & 37 & $\geq 3600$ & 261840105 \\
\hline hamming6-4 & $6^{*}$ & 0 & 4709 & $8^{*}$ & 1 & 71069 & $10^{*}$ & 9 & 849851 \\
\hline hamming8-2 & 128 & $\geq 3600$ & 39716014 & 128 & $\geq 3600$ & 43138327 & 128 & $\geq 3600$ & 50079738 \\
\hline hamming8-4 & 16 & $\geq 3600$ & 237558610 & 18 & $\geq 3600$ & 222683938 & 22 & $\geq 3600$ & 230542048 \\
\hline hamming10-2 & 512 & $\geq 3600$ & 3595516 & 512 & $\geq 3600$ & 3790553 & 512 & $\geq 3600$ & 3877853 \\
\hline hamming $10-4$ & 32 & $\geq 3600$ & 146893539 & 43 & $\geq 3600$ & 132802297 & 64 & $\geq 3600$ & 54961531 \\
\hline johnson8-2-4 & $5^{*}$ & 0 & 1666 & $8^{*}$ & 0 & 12837 & $9^{*}$ & 0 & 104984 \\
\hline johnson8-4-4 & $14^{*}$ & 110 & 11542436 & 18 & $\geq 3600$ & 350491163 & 22 & $\geq 3600$ & 342248079 \\
\hline keller4 & 15 & $\geq 3600$ & 247583422 & 20 & $\geq 3600$ & 207711375 & 22 & $\geq 3600$ & 258859895 \\
\hline MANN-a9 & $26^{*}$ & 66 & 5585820 & $36^{*}$ & 2 & 106834 & $36^{*}$ & 278 & 25470013 \\
\hline MANN-a27 & 234 & $\geq 3600$ & 79044110 & 351 & $\geq 3600$ & 7146812 & 351 & $\geq 3600$ & 10158168 \\
\hline MANN-a45 & 660 & $\geq 3600$ & 19339018 & 990 & $\geq 3600$ & 1022834 & 990 & $\geq 3600$ & 1283088 \\
\hline p-hat300-1 & $10^{*}$ & 14 & 665249 & $12^{*}$ & $\overline{1} 111$ & 40704167 & 14 & $\geq 3600$ & 128042727 \\
\hline p-hat300-2 & 29 & $\geq 3600$ & 167764775 & 35 & $\geq 3600$ & 162883168 & 41 & $\geq 3600$ & 154569677 \\
\hline p-hat300-3 & 42 & $\geq 3600$ & 145501695 & 51 & $\geq 3600$ & 145528614 & 57 & $\geq 3600$ & 139965092 \\
\hline p-hat700-1 & $13^{*}$ & $\overline{1} 887$ & 55769755 & 14 & $\geq 3600$ & 110462323 & 16 & $\geq 3600$ & 98797915 \\
\hline p-hat700-2 & 49 & $\geq 3600$ & 116066244 & 58 & $\geq 3600$ & 116785628 & 65 & $\geq 3600$ & 117405227 \\
\hline p-hat700-3 & 69 & $\geq 3600$ & 105454118 & 81 & $\geq 3600$ & 105553105 & 93 & $\geq 3600$ & 97553599 \\
\hline $\operatorname{san} 200-0.7-2$ & 24 & $\geq 3600$ & 441219398 & 36 & $\geq 3600$ & 395072520 & 48 & $\geq 3600$ & 330336652 \\
\hline $\operatorname{san} 200-0.9-1$ & 90 & $\geq 3600$ & 107493877 & 125 & $\geq 3600$ & 35590748 & 125 & $\geq 3600$ & 39843163 \\
\hline COMP-GEOM-0 & 21 & $\geq 3600$ & 8346 & 22 & $\geq 3600$ & 4672 & 22 & $\geq 3600$ & 6269 \\
\hline COMP-GEOM-1 & 10 & $\geq 3600$ & 754265 & 11 & $\geq 3600$ & 313457 & 12 & $\geq 3600$ & 21132 \\
\hline COMP-GEOM-2 & 8 & $\geq 3600$ & 647635 & 10 & $\geq 3600$ & 423436 & 11 & $\geq 3600$ & 8674 \\
\hline ERDOS-97-1 & $8^{*}$ & 10 & 1390 & $9^{*}$ & 1122 & 198148 & 4 & $\geq 3600$ & 339906 \\
\hline ERDOS-97-2 & 3 & $\geq 3600$ & 737 & 3 & $\geq 3600$ & 480 & 4 & $\geq 3600$ & 470 \\
\hline ERDOS-98-1 & $8^{*}$ & 9 & 1424 & $9^{*}$ & 1250 & 204612 & 4 & $\geq 3600$ & 303239 \\
\hline ERDOS-98-2 & 3 & $\geq 3600$ & 650 & 3 & $\geq 3600$ & 420 & 4 & $\geq 3600$ & 410 \\
\hline ERDOS-99-1 & $8^{*}$ & 10 & 1463 & 9* & 1323 & 211967 & 4 & $\geq 3600$ & 292341 \\
\hline ERDOS-99-2 & 3 & $\geq 3600$ & 591 & 3 & $\geq 3600$ & 376 & 4 & $\geq 3600$ & 369 \\
\hline
\end{tabular}

* optimal 
potential to prune a larger portion of the search tree because $\chi(G[U]) \leq|U|$. Figure 7 shows an algorithm which uses co- $k$-plex coloring to prune the search tree.

Let $k$-plex1a and $k$-plex1b denote the functions obtained by using ICCH and FCCH, respectively, to execute Line 2 of $k$-plex1. ICCH and FCCH are discussed in Section 2. To find a maximum $k$-plex in $G$, run LB1 to find an initial value for max and make the function call to $k$-plex $1 \mathbf{a}(V, \varnothing)$ or $k$-plex $1 \mathbf{b}(V, \varnothing)$. Tables 6 and 7 contain computational results obtained by running these algorithms on the benchmark graphs with a one hour time limit.

\subsection{Algorithm Type 2}

The algorithm in this subsection is based on the following idea of Östergård (2002). Let $V=\left\{v_{1}, \ldots, v_{n}\right\}$ and $S_{i}=\left\{v_{i}, \ldots, v_{n}\right\}$. The algorithm in Figure 5 searches for the largest clique in $S_{1}$ containing $v_{1}$, the largest clique in $S_{2}$ containing $v_{2}$, and so on. Östergård suggests reversing this order. That is, search $S_{n}$ for the largest clique containing $v_{n}, S_{n-1}$ for the largest clique containing $v_{n-1}$, and so on. Let $c(i)$ be the size of the largest clique in $S_{i}$. Clearly, $c(n)=1$ and $c(1)=\omega(G)$. Moreover, $c(i) \in\{c(i+1), c(i+1)+1\}$ for $i=1, \ldots, n-1$.

Figure 8 shows Östergård's clique algorithm. The search order allows for the following pruning strategy. Given candidate set $U$, let $i=\min \left\{j: v_{j} \in U\right\}$. Notice that $U \subseteq S_{i}$ and hence $\omega_{k}(G[U]) \leq c(i)$. This new bound is used in Line 10 in Figure 8 .

Östergård's algorithm adapts to find maximum $k$-plexes with two modifications. First,

\section{function basicPlex $(U, K)$}

1. while $U \neq \varnothing$

2. $\quad$ if $|K|+|U| \leq \max$

3. return

4. $\quad$ end

5. $K=K \cup\{v\} ; \quad U=U \backslash\{v\}$ for some $v \in U$

6. $U^{\prime}:=\{u \in U: K \cup\{u\}$ is a $k$-plex $\}$

7. $\operatorname{basicPlex}\left(U^{\prime}, K\right)$

8. end

9. if $|K|>\max$

10. $\max =|K|$

11. end

12. return

Figure 6: Basic k-plex Algorithm 
Table 6: $k$-plex1a Results

\begin{tabular}{|c|c|c|c|c|c|c|c|c|c|}
\hline$G$ & $\omega_{2}(G)$ & seconds & BBN & $\omega_{3}(G)$ & seconds & BBN & $\omega_{4}(G)$ & seconds & $\mathrm{BBN}$ \\
\hline brock200-1 & 25 & $\geq 3600$ & 86030174 & 28 & $\geq 3600$ & 95147581 & 31 & $\geq 3600$ & 97664910 \\
\hline brock200-2 & $13^{*}$ & 289 & 8663613 & 15 & $\geq 3600$ & 100542983 & 17 & $\geq 3600$ & 90143057 \\
\hline brock200-4 & 19 & $\geq 3600$ & 102282008 & 22 & $\geq 3600$ & 106907189 & 25 & $\geq 3600$ & 98966956 \\
\hline brock $400-2$ & 27 & $\geq 3600$ & 95110220 & 31 & $\geq 3600$ & 88619566 & 35 & $\geq 3600$ & 81344425 \\
\hline brock400-4 & 33 & $\geq 3600$ & 51472394 & 33 & $\geq 3600$ & 74724985 & 36 & $\geq 3600$ & 80938337 \\
\hline brock $800-2$ & 23 & $\geq 3600$ & 94857693 & 26 & $\geq 3600$ & 81559009 & 29 & $\geq 3600$ & 71154628 \\
\hline brock800-4 & 23 & $\geq 3600$ & 96369941 & 25 & $\geq 3600$ & 90097273 & 29 & $\geq 3600$ & 72735746 \\
\hline c-fat200-1 & $12^{*}$ & 2 & 873 & $12^{*}$ & 27 & 57730 & $12^{*}$ & 922 & 1960167 \\
\hline c-fat $200-2$ & $24^{*}$ & 3 & 5269 & $24^{*}$ & 28 & 109070 & $24^{*}$ & 909 & 3385277 \\
\hline c-fat $200-5$ & 58 & $\geq 3600$ & 19298000 & 58 & $\geq 3600$ & 24247513 & 58 & $\geq 3600$ & 28799458 \\
\hline c-fat500-1 & $14^{*}$ & 32 & 2293 & $14^{*}$ & 1580 & 357420 & 14 & $\geq 3600$ & 545917 \\
\hline c-fat $500-2$ & $26^{*}$ & 33 & 20601 & $26^{*}$ & 1617 & 787649 & 26 & $\geq 3600$ & 1224327 \\
\hline c-fat $500-5$ & 64 & $\geq 3600$ & 14631858 & 64 & $\geq 3600$ & 21078075 & 64 & $\geq 3600$ & 24930408 \\
\hline c-fat500-10 & 126 & $\geq 3600$ & 5104395 & 126 & $\geq 3600$ & 7643111 & 126 & $\geq 3600$ & 9068983 \\
\hline hamming6-2 & $32^{*}$ & 0 & 0 & 32 & $\geq 3600$ & 92535097 & 37 & $\geq 3600$ & 119263227 \\
\hline hamming6-4 & $6^{*}$ & 0 & 3668 & $8^{*}$ & 1 & 59533 & $10^{*}$ & 15 & 701425 \\
\hline hamming8-2 & $128^{*}$ & 0 & 0 & 128 & $\geq 3600$ & 14422543 & 128 & $\geq 3600$ & 20007766 \\
\hline hamming8-4 & 16 & $\geq 3600$ & 158903409 & 18 & $\geq 3600$ & 149846604 & 22 & $\geq 3600$ & 157055208 \\
\hline hamming10-2 & $512^{*}$ & 1 & 0 & 512 & $\geq 3600$ & 434296 & 512 & $\geq 3600$ & 456014 \\
\hline hamming10-4 & 43 & $\geq 3600$ & 44661342 & 64 & $\geq 3600$ & 21254123 & 64 & $\geq 3600$ & 33084666 \\
\hline johnson8-2-4 & $5^{*}$ & 0 & 1585 & $8^{*}$ & 0 & 12378 & $9^{*}$ & 1 & 104804 \\
\hline johnson8-4-4 & $14^{*}$ & 138 & 7755953 & 18 & $\geq 3600$ & 191111049 & 22 & $\geq 3600$ & 172931195 \\
\hline keller4 & 15 & $\geq 3600$ & 147002319 & 20 & $\geq 3600$ & 128108327 & 22 & $\geq 3600$ & 169102280 \\
\hline MANN-a9 & $26^{*}$ & 123 & 4111457 & $36^{*}$ & 6 & 102896 & $36^{*}$ & 739 & 25470013 \\
\hline MANN-a27 & 234 & $\geq 3600$ & 78820556 & 351 & $\geq 3600$ & 383569 & 351 & $\geq 3600$ & 781334 \\
\hline MANN-a45 & 660 & $\geq 3600$ & 18866263 & 990 & $\geq 3600$ & 18029 & 990 & $\geq 3600$ & 53068 \\
\hline p-hat300-1 & $10^{*}$ & 33 & 562727 & $12^{*}$ & 2827 & 39631513 & 14 & $\geq 3600$ & 54618899 \\
\hline p-hat300-2 & 30 & $\geq 3600$ & 77146967 & 35 & $\geq 3600$ & 84162645 & 41 & $\geq 3600$ & 84779804 \\
\hline p-hat300-3 & 42 & $\geq 3600$ & 73906134 & 50 & $\geq 3600$ & 70787196 & 57 & $\geq 3600$ & 70408792 \\
\hline p-hat700-1 & $13^{*}$ & 3186 & 46290951 & 14 & $\geq 3600$ & 56967864 & 16 & $\geq 3600$ & 43437614 \\
\hline p-hat700-2 & 50 & $\geq 3600$ & 51487369 & 58 & $\geq 3600$ & 56760785 & 65 & $\geq 3600$ & 61159187 \\
\hline p-hat700-3 & 70 & $\geq 3600$ & 42921661 & 82 & $\geq 3600$ & 46670755 & 92 & $\geq 3600$ & 48056462 \\
\hline $\operatorname{san} 200-0.7-2$ & 26 & $\geq 3600$ & 247380139 & 36 & $\geq 3600$ & 368232192 & 48 & $\geq 3600$ & 301494773 \\
\hline $\operatorname{san} 200-0.9-1$ & 90 & $\geq 3600$ & 64534714 & 125 & $\geq 3600$ & 5514998 & 125 & $\geq 3600$ & 6899702 \\
\hline COMP-GEOM-0 & $22^{*}$ & 1218 & 3301 & 22 & $\overline{\geq} 3600$ & 18785 & 22 & $\overline{\geq} 3600$ & 173911 \\
\hline COMP-GEOM-1 & $10^{*}$ & 1205 & 2366 & 11 & $\geq 3600$ & 110109 & 11 & $\geq 3600$ & 122167 \\
\hline COMP-GEOM-2 & $8^{*}$ & 1220 & 1211 & 8 & $\geq 3600$ & 14166 & 8 & $\geq 3600$ & 6208 \\
\hline ERDOS-97-1 & $8^{*}$ & 313 & 954 & 9 & $\geq 3600$ & 5742 & 10 & $\geq 3600$ & 5571 \\
\hline ERDOS-97-2 & 8 & $\geq 3600$ & 2698 & 9 & $\geq 3600$ & 1494 & 10 & $\geq 3600$ & 1685 \\
\hline ERDOS-98-1 & $8^{*}$ & 427 & 1016 & 9 & $\geq 3600$ & 6008 & 10 & $\geq 3600$ & 5876 \\
\hline ERDOS-98-2 & 8 & $\geq 3600$ & 2359 & 9 & $\geq 3600$ & 1086 & 10 & $\geq 3600$ & 1771 \\
\hline ERDOS-99-1 & $8^{*}$ & 406 & 472 & 9 & $\geq 3600$ & 6248 & 10 & $\geq 3600$ & 5135 \\
\hline ERDOS-99-2 & 8 & $\geq 3600$ & 2913 & 9 & $\geq 3600$ & 943 & 10 & $\geq 3600$ & 1544 \\
\hline
\end{tabular}


Table 7: $k$-plex1b Results

\begin{tabular}{|c|c|c|c|c|c|c|c|c|c|}
\hline$G$ & $\omega_{2}(G)$ & seconds & $\mathrm{BBN}$ & $\omega_{3}(G)$ & seconds & $\mathrm{BBN}$ & $\omega_{4}(G)$ & seconds & $\mathrm{BBN}$ \\
\hline brock200-1 & 25 & $\geq 3600$ & 10054924 & 28 & $\geq 3600$ & 9610060 & 31 & $\geq 3600$ & 11595841 \\
\hline brock200-2 & $13^{*}$ & 1778 & 7722362 & 15 & $\geq 3600$ & 15728716 & 17 & $\geq 3600$ & 15883075 \\
\hline brock200-4 & 19 & $\geq 3600$ & 12056663 & 22 & $\geq 3600$ & 11842251 & 24 & $\geq 3600$ & 13308843 \\
\hline brock400-2 & 27 & $\geq 3600$ & 3298972 & 31 & $\geq 3600$ & 2506819 & 35 & $\geq 3600$ & 3084792 \\
\hline brock400-4 & 33 & $\geq 3600$ & 2879018 & 33 & $\geq 3600$ & 2769768 & 36 & $\geq 3600$ & 3339363 \\
\hline brock800-2 & 22 & $\geq 3600$ & 847946 & 26 & $\geq 3600$ & 687075 & 29 & $\geq 3600$ & 719475 \\
\hline brock800-4 & 22 & $\geq 3600$ & 846155 & 25 & $\geq 3600$ & 708185 & 29 & $\geq 3600$ & 771036 \\
\hline c-fat200-1 & $12^{*}$ & 3 & 802 & $12^{*}$ & 41 & 57612 & $12^{*}$ & 1397 & 1958425 \\
\hline c-fat $200-2$ & $24^{*}$ & 3 & 2050 & $24^{*}$ & 60 & 96754 & 24 & $\geq 3600$ & 1586893 \\
\hline c-fat200-5 & $58^{*}$ & 836 & 444241 & 58 & $\geq 3600$ & 3960477 & 58 & $\geq 3600$ & 5319030 \\
\hline c-fat500-1 & $14^{*}$ & 34 & 2060 & $14^{*}$ & 1796 & 356744 & 14 & $\geq 3600$ & 427000 \\
\hline c-fat500-2 & $26^{*}$ & 39 & 10177 & $26^{*}$ & 2368 & 754350 & 26 & $\geq 3600$ & 797974 \\
\hline c-fat500-5 & $64^{*}$ & 1579 & 557081 & 64 & $\geq 3600$ & 2800459 & 64 & $\geq 3600$ & 3453142 \\
\hline c-fat500-10 & 126 & $\geq 3600$ & 327032 & 126 & $\geq 3600$ & 974543 & 126 & $\geq 3600$ & 1193712 \\
\hline hamming6-2 & $32^{*}$ & 0 & 0 & 32 & $\geq 3600$ & 6160422 & 36 & $\geq 3600$ & 44987310 \\
\hline hamming6-4 & $6^{*}$ & 0 & 3380 & $8^{*}$ & 3 & 58663 & $10^{*}$ & 33 & 693982 \\
\hline hamming8-2 & $128^{*}$ & 0 & 0 & 128 & $\geq 3600$ & 636791 & 128 & $\geq 3600$ & 2835717 \\
\hline hamming8-4 & 16 & $\geq 3600$ & 9945892 & 18 & $\geq 3600$ & 9748498 & 18 & $\geq 3600$ & 10199713 \\
\hline hamming10-2 & $512^{*}$ & 1 & 0 & 512 & $\geq 3600$ & 7508 & 512 & $\geq 3600$ & 29602 \\
\hline hamming10-4 & 43 & $\geq 3600$ & 323816 & 64 & $\geq 3600$ & 370717 & 64 & $\geq 3600$ & 236250 \\
\hline johnson8-2-4 & $5^{*}$ & 0 & 1585 & $8^{*}$ & 0 & 12337 & $9 *$ & 2 & 104679 \\
\hline johnson8-4-4 & $14^{*}$ & 475 & 6389736 & 18 & $\geq 3600$ & 48544486 & 22 & $\geq 3600$ & 52307238 \\
\hline keller4 & 15 & $\geq 3600$ & 18263136 & 20 & $\geq 3600$ & 17714412 & 22 & $\geq 3600$ & 20281149 \\
\hline MANN-a9 & $26^{*}$ & 395 & 3240597 & $36^{*}$ & 15 & 100969 & $36^{*}$ & 1733 & 25470013 \\
\hline MANN-a27 & 234 & $\geq 3600$ & 3053292 & 351 & $\geq 3600$ & 78758 & 351 & $\geq 3600$ & 253074 \\
\hline MANN-a45 & 660 & $\geq 3600$ & 28446 & 990 & $\geq 3600$ & 7035 & 990 & $\geq 3600$ & 20406 \\
\hline p-hat300-1 & $10^{*}$ & 139 & 500766 & 12 & $\geq 3600$ & 12816637 & 14 & $\geq 3600$ & 12577276 \\
\hline p-hat300-2 & 30 & $\geq 3600$ & 7335988 & 35 & $\geq 3600$ & 6439794 & 40 & $\geq 3600$ & 8083792 \\
\hline p-hat300-3 & 42 & $\geq 3600$ & 5279160 & 50 & $\geq 3600$ & 5693325 & 57 & $\geq 3600$ & 5957872 \\
\hline p-hat700-1 & 12 & $\geq 3600$ & 2617164 & 13 & $\geq 3600$ & 2557821 & 16 & $\geq 3600$ & 2516929 \\
\hline p-hat700-2 & 50 & $\geq 3600$ & 1176955 & 58 & $\geq 3600$ & 1407568 & 65 & $\geq 3600$ & 1459008 \\
\hline p-hat700-3 & 70 & $\geq 3600$ & 750652 & 82 & $\geq 3600$ & 1007772 & 92 & $\geq 3600$ & 1001573 \\
\hline $\operatorname{san} 200-0.7-2$ & 26 & $\geq 3600$ & 12473403 & 36 & $\geq 3600$ & 16047112 & 48 & $\geq 3600$ & 16009322 \\
\hline san200-0.9-1 & 90 & $\geq 3600$ & 9748246 & 125 & $\geq 3600$ & 970390 & 125 & $\geq 3600$ & 1705032 \\
\hline COMP-GEOM-0 & $22^{*}$ & 2202 & 2797 & 22 & $\geq 3600$ & 15368 & 22 & $\geq 3600$ & 71325 \\
\hline COMP-GEOM-1 & $10^{*}$ & 2075 & 2013 & 11 & $\geq 3600$ & 10073 & 11 & $\geq 3600$ & 20237 \\
\hline COMP-GEOM-2 & $8^{*}$ & 2731 & 1193 & 8 & $\geq 3600$ & 4102 & 8 & $\geq 3600$ & 6028 \\
\hline ERDOS-97-1 & $8^{*}$ & 370 & 869 & 9 & $\geq 3600$ & 4928 & 10 & $\geq 3600$ & 4961 \\
\hline ERDOS-97-2 & 8 & $\geq 3600$ & 574 & 9 & $\geq 3600$ & 702 & 10 & $\geq 3600$ & 671 \\
\hline ERDOS-98-1 & $8^{*}$ & 414 & 953 & 9 & $\geq 3600$ & 4497 & 10 & $\geq 3600$ & 4506 \\
\hline ERDOS-98-2 & 8 & $\geq 3600$ & 449 & 9 & $\geq 3600$ & 492 & 10 & $\geq 3600$ & 487 \\
\hline ERDOS-99-1 & $8^{*}$ & 438 & 989 & 9 & $\geq 3600$ & 4379 & 10 & $\geq 3600$ & 4376 \\
\hline ERDOS-99-2 & 8 & $\geq 3600$ & 592 & 9 & $\geq 3600$ & 633 & 10 & $\geq 3600$ & 615 \\
\hline
\end{tabular}




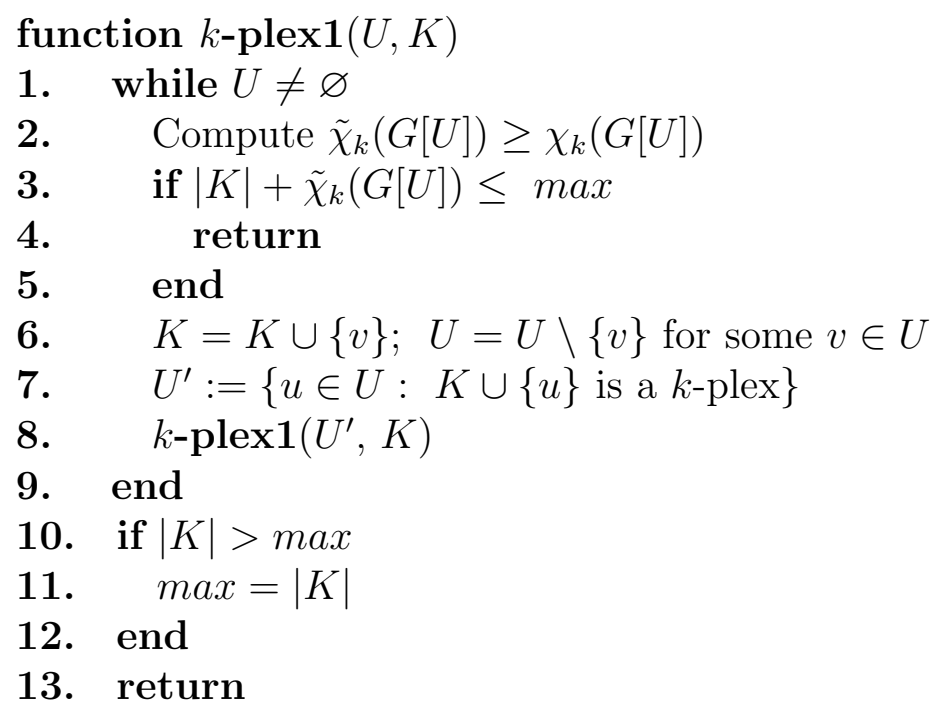

Figure 7: $k$-plex Algorithm

define $c_{k}(i)=\omega_{k}\left(G\left[S_{i}\right]\right)$. Second, the candidate set with respect to $K$ is defined as

$$
U:=\{v \in V \backslash K: K \cup\{v\} \text { is a } k \text {-plex }\} .
$$

Figure 9 shows $k$-plex2. Table 8 contains computational results obtained by running $k$ plex2 on the benchmark graphs with a one hour time limit.

\section{Conclusions and Future Work}

This paper describes combinatorial algorithms for finding maximum $k$-plexes in a graph. Section 2 focuses on co-k-plex coloring heuristics which are used as an upper bound on the $k$-plex number. Section 3 discusses a heuristic for finding maximum $k$-plexes. This heuristic provides a lower bound on the $k$-plex number. Section 4 describes exact algorithms for finding maximum $k$-plexes. Table 9 summarizes the number of instances solved to optimality by each exact algorithm.

The first three exact algorithms perform similarly within the hour time limit. Although this type of algorithm appears to benefit from the upper and lower bound heuristics, they solve a relatively small number of instances to optimality. This suggests that the coloring heuristics might not produce tight upper bounds, so an interesting avenue for future work would be to develop stronger coloring heuristics. 
function OsterClique $(U, K)$

1. if $|U|=0$

2. if $|K|>\max$

3. $\max =|K|$

4. found =true

5. end

6. return

7. end

8. while $U \neq \varnothing$

9. if $|K|+|U| \leq \max$

10. return

11. end

12. $i=\min \left\{j: v_{j} \in U\right\}$

13. if $|K|+c(i) \leq \max$

14. return

15. end

16. $U=U \backslash\left\{v_{i}\right\}$

17. OsterClique $\left(U \cap N_{G}\left(v_{i}\right), K \cup\left\{v_{i}\right\}\right)$

18. if found=true

19. return

20. end

21. end

22. return

function findClique

23. $\max =0$

24. for $i=n$ down to 1

25. found $=$ false

26. OsterClique $\left(S_{i} \cap N_{G}\left(v_{i}\right),\left\{v_{i}\right\}\right)$

27. end

28. $c(i)=\max$

29. return

Figure 8: Östergård's Clique Algorithm 


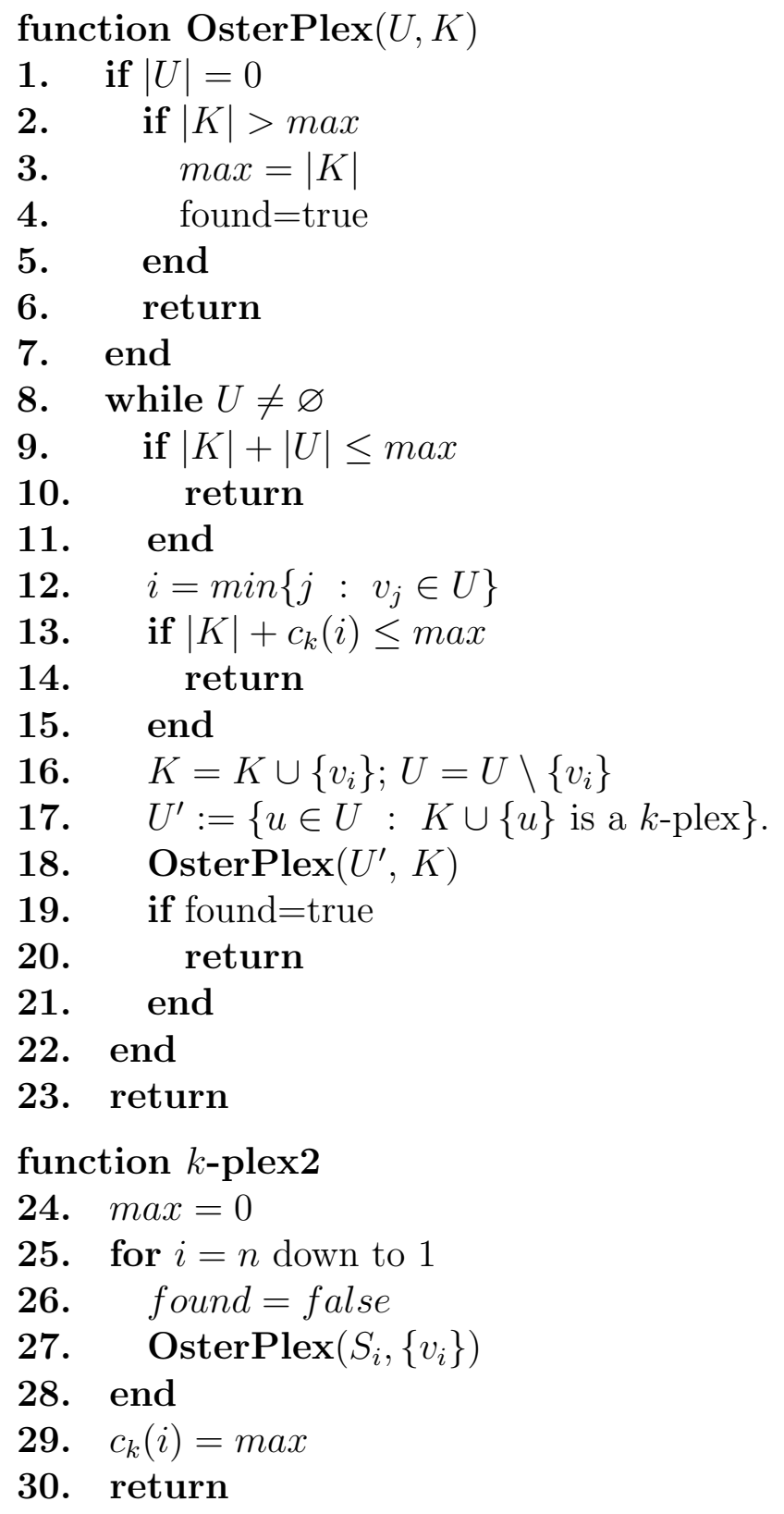

Figure 9: Östergård's Algorithm Adapted for $k$-plexes

A natural approach for improving the co- $k$-plex coloring heuristics would be to generalize the DSATUR graph coloring heuristic (Brélaz's 1979). The DSATUR heuristic dynamically determines the order in which vertices are colored. More precisely, it maintains the number of distinct colors adjacent to each uncolored vertex and always colors the vertex with the highest number of adjacent color classes. The number of adjacent colors classes defines a vertex's saturation degree. This idea generalizes to co- $k$-plex coloring. The saturation degree of $v$ is redefined as the number of distinct partition sets $C_{i}$ such that $C_{i} \cup\{v\}$ is not a 
Table 8: $k$-plex2 Results

\begin{tabular}{|c|c|c|c|c|c|c|c|c|c|}
\hline$G$ & $\omega_{2}(G)$ & seconds & $\mathrm{BBN}$ & $\omega_{3}(G)$ & seconds & $\mathrm{BBN}$ & $\omega_{4}(G)$ & seconds & $\mathrm{BBN}$ \\
\hline brock200-1 & 23 & $\geq 3600$ & 983266826 & 24 & $\geq 3600$ & 1061715139 & 27 & $\geq 3600$ & 1090413176 \\
\hline brock200-2 & $13^{*}$ & 74 & 19636408 & 15 & $\geq 3600$ & 973470713 & 17 & $\geq 3600$ & 1118582507 \\
\hline brock200-4 & 19 & $\geq 3600$ & 917681365 & 20 & $\geq 3600$ & 1078546865 & 21 & $\geq 3600$ & 1158926695 \\
\hline brock $400-2$ & 23 & $\geq 3600$ & 996101972 & 27 & $\geq 3600$ & 1112675498 & 29 & $\geq 3600$ & 1152442843 \\
\hline brock400-4 & 23 & $\geq 3600$ & 989222371 & 22 & $\geq 3600$ & 1132833870 & 30 & $\geq 3600$ & 1159027166 \\
\hline brock800-2 & 21 & $\geq 3600$ & 926436143 & 20 & $\geq 3600$ & 1077097050 & 23 & $\geq 3600$ & 1142792112 \\
\hline brock800-4 & 20 & $\geq 3600$ & 919220732 & 22 & $\geq 3600$ & 1044131631 & 24 & $\geq 3600$ & 1109841414 \\
\hline c-fat200-1 & $12^{*}$ & 0 & 3677 & $12^{*}$ & 0 & 123687 & $12^{*}$ & 19 & 4452622 \\
\hline c-fat $200-2$ & $24^{*}$ & 0 & 1895 & $24^{*}$ & 0 & 30227 & $24^{*}$ & 2 & 759423 \\
\hline c-fat $200-5$ & $58^{*}$ & 0 & 760 & $58^{*}$ & 0 & 6566 & $58^{*}$ & 0 & 88301 \\
\hline c-fat500-1 & $14^{*}$ & 0 & 19733 & $14^{*}$ & 8 & 1183127 & $14^{*}$ & 1416 & 134845416 \\
\hline c-fat $500-2$ & $26^{*}$ & 0 & 10081 & $26^{*}$ & 2 & 266957 & $26^{*}$ & 91 & 12812559 \\
\hline c-fat500-5 & $64^{*}$ & 0 & 4195 & $64^{*}$ & 0 & 52676 & $64^{*}$ & 6 & 956345 \\
\hline c-fat500-10 & $126^{*}$ & 0 & 2141 & $126^{*}$ & 0 & 18391 & $126^{*}$ & 2 & 221427 \\
\hline hamming6-2 & $32^{*}$ & 0 & 396 & $32^{*}$ & 1 & 195054 & $40^{*}$ & 872 & 226208834 \\
\hline hamming6-4 & $6^{*}$ & 0 & 4526 & $8^{*}$ & 0 & 37113 & $10^{*}$ & 1 & 395729 \\
\hline hamming8-2 & $128^{*}$ & 0 & 7448 & $128^{*}$ & 2744 & 182864232 & 112 & $\geq 3600$ & 808066329 \\
\hline hamming8-4 & $16^{*}$ & 47 & 7982728 & 17 & $\geq 3600$ & 1053193592 & 19 & $\geq 3600$ & 1045849871 \\
\hline hamming10-2 & $512^{*}$ & 33 & 147919 & 448 & $\geq 3600$ & 208033053 & 430 & $\geq 3600$ & 799045131 \\
\hline hamming10-4 & 41 & $\geq 3600$ & 595181620 & 46 & $\geq 3600$ & 1058346589 & 51 & $\geq 3600$ & 1042679457 \\
\hline johnson8-2-4 & $5^{*}$ & 0 & 2621 & $8^{*}$ & 0 & 10472 & $9^{*}$ & 0 & 151028 \\
\hline johnson8-4-4 & $14^{*}$ & 0 & 40896 & $18^{*}$ & 39 & 14014988 & 21 & $\geq 3600$ & 1160638581 \\
\hline keller4 & $15^{*}$ & 1000 & 284120617 & 21 & $\geq 3600$ & 909167108 & 16 & $\geq 3600$ & 1183954679 \\
\hline MANN-a9 & $26^{*}$ & 0 & 53102 & $36^{*}$ & 1 & 375502 & $36^{*}$ & 129 & 36511315 \\
\hline MANN-a27 & 235 & $\geq 3600$ & 33275514 & 351 & $\geq 3600$ & 23112644 & 351 & $\geq 3600$ & 93317993 \\
\hline MANN-a 45 & 661 & $\geq 3600$ & 3326395 & 990 & $\geq 3600$ & 2840051 & 990 & $\geq 3600$ & 16526869 \\
\hline p-hat300-1 & $10^{*}$ & 6 & 1561119 & $12^{*}$ & 552 & 128854637 & 13 & $\geq 3600$ & 1001501766 \\
\hline p-hat300-2 & 29 & $\geq 3600$ & 704387512 & 29 & $\geq 3600$ & 909116307 & 33 & $\geq 3600$ & 987023800 \\
\hline p-hat300-3 & 34 & $\geq 3600$ & 850772303 & 37 & $\geq 3600$ & 956121775 & 36 & $\geq 3600$ & 1050444550 \\
\hline p-hat700-1 & $13^{*}$ & 667 & 90760266 & 13 & $\geq 3600$ & 687249461 & 13 & $\geq 3600$ & 964257683 \\
\hline p-hat700-2 & 37 & $\geq 3600$ & 655931022 & 39 & $\geq 3600$ & 804657901 & 42 & $\geq 3600$ & 937295855 \\
\hline p-hat700-3 & 51 & $\geq 3600$ & 820473217 & 43 & $\geq 3600$ & 991432587 & 44 & $\geq 3600$ & 1063865458 \\
\hline $\operatorname{san} 200-0.7-2$ & 24 & $\geq 3600$ & 1079696544 & 36 & $\geq 3600$ & 940350668 & 48 & $\geq 3600$ & 862621323 \\
\hline $\operatorname{san} 200-0.9-1$ & 90 & $\geq 3600$ & 485362621 & $125^{*}$ & 253 & 64534321 & 50 & $\geq 3600$ & 1006342152 \\
\hline COMP-GEOM-0 & $22^{*}$ & 397 & 2150878 & 5 & $\geq 3600$ & 156104820 & 4 & $\geq 3600$ & 265372393 \\
\hline COMP-GEOM-1 & $10^{*}$ & 1118 & 4875730 & 4 & $\geq 3600$ & 172334737 & 4 & $\geq 3600$ & 267076112 \\
\hline COMP-GEOM-2 & $8^{*}$ & 1145 & 5034335 & 4 & $\geq 3600$ & 162641029 & 4 & $\geq 3600$ & 266562165 \\
\hline ERDOS-97-1 & $8^{*}$ & 0 & 25770 & $9^{*}$ & 19 & 2391684 & $11^{*}$ & 1897 & 256331821 \\
\hline ERDOS-97-2 & $8^{*}$ & 1253 & 12602187 & 3 & $\geq 3600$ & 216016439 & 4 & $\geq 3600$ & 331758265 \\
\hline ERDOS-98-1 & $8^{*}$ & 0 & 27246 & $9^{*}$ & 20 & 2552046 & $11^{*}$ & 1675 & 212106816 \\
\hline ERDOS-98-2 & $8^{*}$ & 1514 & 14265207 & 3 & $\geq 3600$ & 209965048 & 4 & $\geq 3600$ & 318616472 \\
\hline ERDOS-99-1 & $8^{*}$ & 0 & 28035 & $9 *$ & 21 & 2632545 & $11^{*}$ & 1783 & 223030325 \\
\hline ERDOS-99-2 & $8^{*}$ & 1757 & 15748963 & 3 & $\geq 3600$ & 205078469 & 4 & $\geq 3600$ & 308498817 \\
\hline
\end{tabular}

* optimal

co- $k$-plex. This general version of DSATUR was implemented and tested (McClosky 2008). However, the results did not improve upon ICCH and FCCH.

The final exact algorithm, $k$-plex2, dominates the Type 1 algorithms with respect to number of instances solved to optimality. Moreover, $k$-plex2 converges quickly, when it converges at all. However, the final solution can be far from optimal when $k$-plex2 fails to converge. The algorithm demonstrates this phenomenon on the collaboration networks for $k=3,4$. The reason for this behavior is that the algorithm can spend much of its time optimizing over a subset of $V$. Consequently, if vertices at the end of the vertex ordering are needed to make large $k$-plexes, good solutions cannot be found until the entire vertex set is 
Table 9: Results Summary

\begin{tabular}{lcccc}
\hline Algorithm & $k=2$ & $k=3$ & $k=4$ & Total \\
\hline basicPlex & 16 & 11 & 5 & 32 \\
$k$-plex1a & 20 & 8 & 5 & 33 \\
$k$-plex1b & 21 & 7 & 4 & 32 \\
$k$-plex2 & 28 & 18 & 14 & 60 \\
\hline
\end{tabular}

processed. This illustrates the importance of vertex ordering for this type of algorithm.

Type 1 algorithms spend time at each branch and bound node to approximate $\chi_{k}(G[U])$ for the candidate set $U$. Unfortunately, $\chi_{k}(G)$ could be an inaccurate bound on $\omega_{k}(G)$ in general. The $k$-plex2 algorithm spends no time estimating $\chi_{k}(G)$ but benefits from the bound obtained using the $c_{k}$ array. In any case, the purpose of these bounds is to prune the candidate set. The requirement for membership in the candidate set becomes less stringent as $k$ increases, so the set becomes harder to prune. This contributes to the increase in runtime as $k$ grows.

When comparing these results to those found in Balasundaram et al. (2006), the combinatorial algorithms outperform branch-and-cut on the DIMACS graphs. On the other hand, branch-and-cut works better on the larger social network graphs. This suggests that combinatorial methods are superior for graphs on a few hundred vertices, but branch-and-cut becomes the preferred method as the size of the graphs grow.

Another area for future research is an exact co- $k$-plex coloring algorithm. The co- $k$-plex chromatic number is unknown for most of the benchmark graphs. Therefore, it is difficult to evaluate the performance of the co-k-plex coloring heuristics in Section 2. It would also be beneficial to study additional heuristics for both the upper and lower bounds on $\omega_{k}(G)$.

\section{Acknowledgments}

This research was partially supported by NSF grants DMI-0521209 and DMS-0729251. Svyatoslav Trukhanov has independently generalized Östergård's clique algorithm to find maximum $k$-plexes (Trukhanov 2008).

\section{References}

Applegate, D. and Johnson, D. 1993. dfmax.c. ftp://dimacs.rutgers.edu/pub/challange/graph/solvers/. 
Atamtürk, A., Nemhauser, G.L., and Savelsbergh, M.W.P. 2000. Conflict graphs in solving integer programming problems. European Journal of Operational Research 121 40-55.

Balas, E. and Xue, J. 1996. Weighted and unweighted maximum clique algorithms with upper bounds from fractional coloring. Algorithmica 15 397-412.

Balasundaram, B., Butenko, S., Hicks, I.V. and Sachdeva, S. 2006. Clique relaxations in social network analysis: The maximum $k$-plex problem. Submitted.

V. Batagelj and A. Mrvar, 2006. Pajek datasets. http://vlado.fmf.uni-lj.si/pub/networks/data/, 2006.

Beebe, N.H.F. (2002): Nelson H.F. Beebe's Bibliographies Page.

Butenko, S. and Wilhelm, W. 2006. Clique-detection models in computational biochemistry and genomics. European Journal of Operational Research, 173 1-17.

Carraghan, R. and Pardalos, P.M. 1990. An exact algorithm for the maximum clique problem. Oper Res. Lett. 9 375-382.

Chen, Y. P., Liestman, A. L., and Liu, J. 2004. Clustering algorithms for ad hoc wireless networks. Ad Hoc and Sensor Networks (Y. Pan and Y. Xiao eds.), Nova Science Publishers.

DIMACS. Cliques, Coloring, and Satisability: Second DIMACS Implementation Challenge. http://dimacs.rutgers.edu/Challenges/, 1995. Accessed 2006.

Festinger, L. 1949. The analysis of sociograms using matrix algebra. Human Relations 10:153-58.

Friedkin, N.E.1984. Structural cohesion and equivalence explanations of social homogeneity. Sociological Methods \& Research 12: 235-261.

Jones, B., Computational Geometry Database, February 2002; FTP / HTTP

Luce, R. and Perry, A. 1949. A method of matrix analysis of group structure. Psychometrika 14, 95-116.

McClosky, B. and Hicks, I. V. 2007. The co-2-plex polytope and integral systems, SIAM Journal of Discrete Mathematics, to appear.

McClosky, B. 2008. Independence Systems and Stable Set Relaxations. Ph.D. thesis, Computational and Applied Mathematics Department, Rice University, Houston, TX.

Moon, J.W. and Moser, L. 1965. On cliques in graphs. Israel J. Math. 3 23-28. 
Östergård, P. R. J. 2002. A fast algorithm for the maximum clique problem. Discrete Appl. Math. 120 197-207.

V. Batagelj and A. Mrvar, 2006. Pajek datasets. http://vlado.fmf.uni-lj.si/pub/networks/data/. Accessed 2006.

Régin, J.C. 2003. Solving the maximum clique problem with constraint programming. Fifth International Workshop on Integration of AI and OR Techniques in Constraint Programming for Combinatorial Optimization Problems. 166-179.

Seidman, S. B. and Foster, B. L. 1978. A graph theoretic generalization of the clique concept. Journal of Mathematical Sociology 6 139-154.

Sewell, E.C. 1998. A branch and bound algorithm for the stability number of a sparse graph. INFORMS J. Comput. 10 438-447.

Tomita, E. and Seki, T. 2003. An efficient branch-and-bound algorithm for finding a maximum clique. Lecture Notes in Computer Science Series 2731 278-289.

Trukhanov, S. 2008, personal communication.

Washio, T. and Motoda, H. 2003. State of the art of graph-based data mining. SIGKDD Explor. Newsl.. 5(1) 59-68.

Wasserman, S. and Faust, K. 1994. Social Network Analysis, Cambridge University Press.

Wood, D.R. 1997. An algorithm for finding a maximum clique in a graph. Oper. Res. Lett. $21211-217$. 